\title{
Notes and Comment
}

\section{A revised table of $d^{\prime}$ for M-alternative forced choice}

\author{
MICHAEL J. HACKER \\ The Rockefeller University \\ New York, New York 10021 \\ and \\ ROGER RATCLIFF \\ Dartmouth College \\ Hanover, New Hampshire 03755
}

The use of $d^{\prime}$ as a dependent measure of manyalternative, forced-choice performance is widespread in the study of perception and memory. Elliot's (1964) tables, which list $d^{\prime}$ as a function of the number of orthogonal alternatives $(\mathrm{M})$ and the proportion of correct choices $(\mathrm{P})$, have become a standard reference (as evidenced by recent citations in undergraduate texts, e.g., Kintsch, 1977; Massaro, 1975). Unfortunately, the tabled values of $d^{\prime}$ are not accurate to the two decimal places listed and occasionally are in error in the first decimal place. The purpose of this note is to provide a revision of Elliot's tables in which the precision has been uniformly extended to two decimal places and the coverage has been expanded to include several more low values of $\mathrm{M}$.

In fairness to Elliot, the original values were never represented as accurate to the nearest hundredth. Although somewhat indirectly stated, $d^{\prime}$ for an arbitrary $M$ alternatives and $P$ proportion correct was warranted only to lie within the interval bounded by the true $\mathrm{d}^{\prime}$ values for expected proportions $(\mathrm{P}-.02)$ and $(P+.02)$. As an illustration with four alternatives and $70 \%$ correct choice, $\mathrm{d}^{\prime}$ could take any value from 1.42 to 1.57 (see Table 1 on the following two pages), although the reported values seldom approach the maximum allowable error. In addition to Elliot's acknowledgment, it is unlikely that the errors in the tabled values have greatly affected any substantive conclusions. Nevertheless, the increased availability of computing facilities since these values first appeared in 1959 (see Elliot, 1964) has reduced our tolerance for imprecision, while the sustained usage of $\mathrm{d}^{\prime}$ as a performance measure suggests the even greater usefulness of an expanded table.

This research was partially supported by NSF Grant BNS 7609959. The first author was further supported by NIMH Training Grant 15125 , and the second author was a visiting assistant professor at Rockefeller University. Requests for reprints should be sent to Michael J. Hacker, who is now at the Department of Psychology and Social Relations, Harvard University, Cambridge, Massachusetts 02138 .
Table 1 was based on numerical evaluation of the expression

$$
P=\int_{-\infty}^{+\infty} \phi\left(x-d^{\prime}\right) \Phi(x)^{M-1} d x .
$$

Given that $\phi$ and $\Phi$ represent the ordinate and area under the lower tail of the unit normal distribution, respectively, the integral is conveniently interpreted as the likelihood of a sample value from the signal distribution exceeding all of the sample values from $\mathbf{M}-1$ identical and independent noise distributions. In order to avoid the difficulties of double integrations, $\Phi(\mathrm{x})$ was approximated to six decimal places by an algebraic formula (Hastings, 1955). The main integral was approximated from $\mathrm{x}=-4.0$ to 10.0 using Simpson's rule with a minimum of 175 intervals. All calculations were in double precision (17 figures on a PDP-11/10). Finally, since Equation 1 is actually an inverse function for present purposes, a hill-climbing routine was employed to search out $d^{\prime}$ values which yielded expected values of $p$ within .0001 of the tabled arguments. ${ }^{1}$

As a precaution against programming errors, $d^{\prime} s$ were also computed via an equivalent expression ${ }^{2}$ proposed by Elliot; viz,

$$
\begin{aligned}
1-P & =\int_{0}^{+\infty} z \exp \left(\mathrm{z}^{2} / 2\right) \\
& \times \Phi\left(\Phi^{-1}\left\{\exp \left[\mathrm{z}^{2} /(2 \mathrm{M}-2)\right]\right\}-\mathrm{d}^{\prime}\right) \mathrm{d} z,
\end{aligned}
$$

where the original limits of integration and number of intervals were appropriately increased to obtain the desired level of accuracy. Finally, we undertook an independent check of selected $d^{\prime} s$ on a separate computer system using Equation 1 with Gaussian quadrature and a different search routine.

\section{REFERENCES}

Elliot, P. B. Tables of $\mathrm{d}^{\prime}$. In J. A. Swets (Ed.), Signal detection and recognition by human observers. New York: Wiley, 1964.

Hastings, C. Approximations for digital computers. Princeton, N.J: Princeton University Press, 1955.

Kintsch, W. Memory and cognition. New York: Wiley, 1977.

Massaro, D. W. Experimental psychology and information processing. Chicago: Rand McNally, 1975.

\section{NOTES}

1. A related FORTRAN program allowing the user to specify nontabled values of $P$ and $M$ is available from the first author.

2. Equation 2 is obtained from Equation 1 following a change of variable. A misprint in Elliot (1964) includes the $-\mathrm{d}^{\prime}$ term with the argument of the inverse cumulative normal, $\Phi^{-1}$.

(Received and accepted for publication June 26, 1979.) 


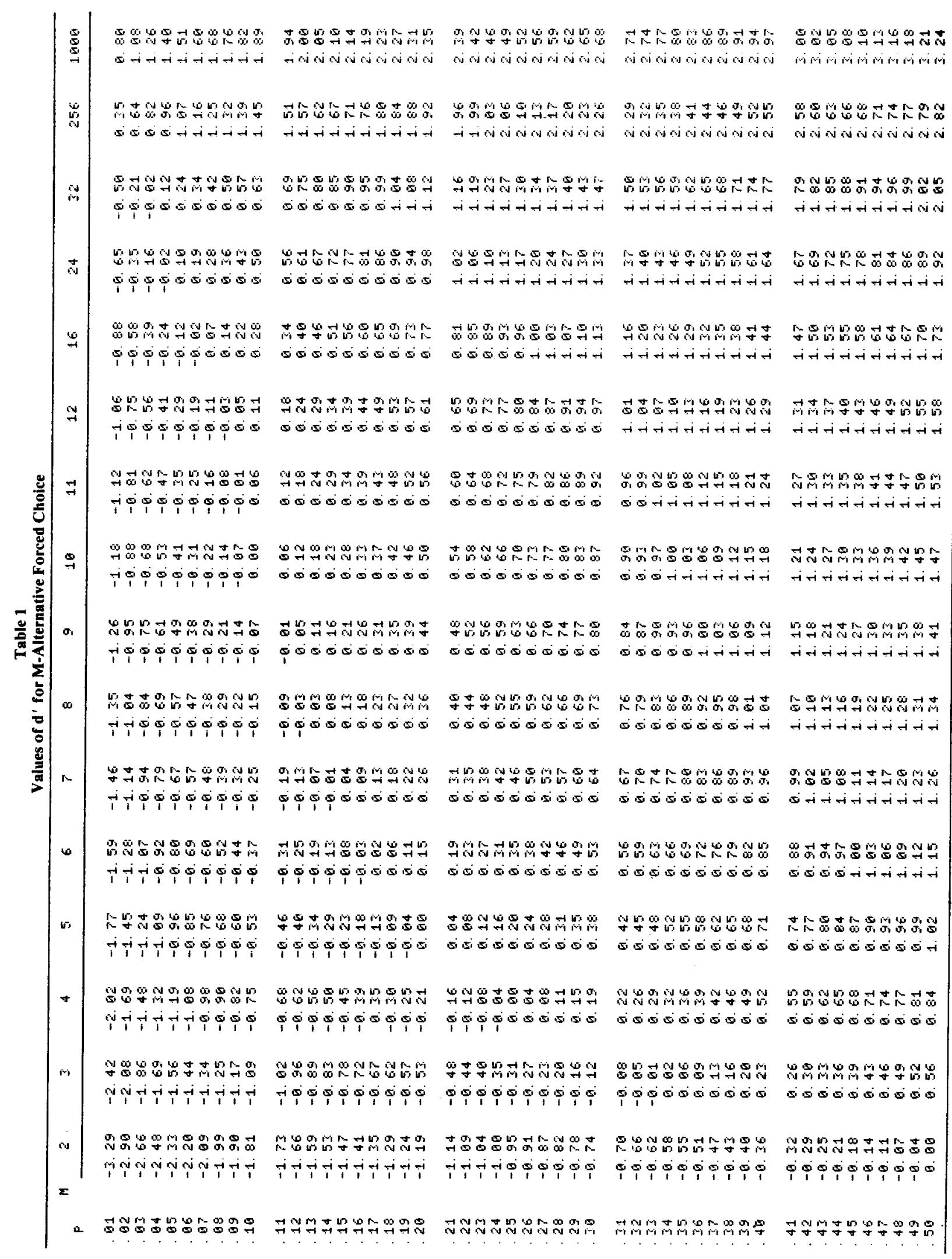




\begin{tabular}{|c|c|c|c|c|c|}
\hline 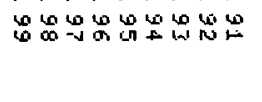 & 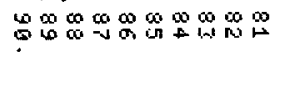 & 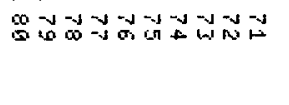 & 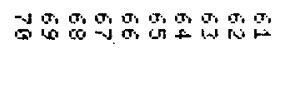 & 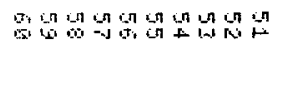 & 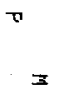 \\
\hline 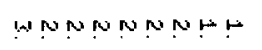 & 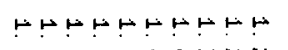 & 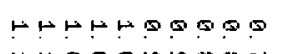 & 900000005 & 500000000 & \\
\hline 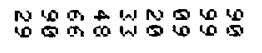 & 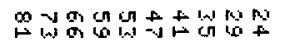 & 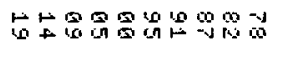 & 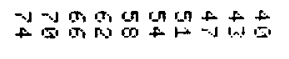 & 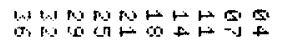 & $\omega$ \\
\hline 舟 & 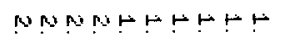 & 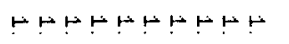 & トトトケトトトののコ & 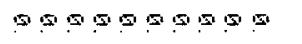 & \\
\hline 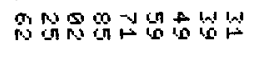 & 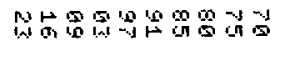 & 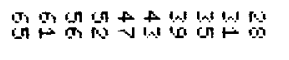 & 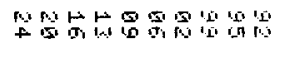 & 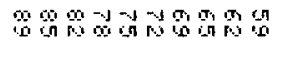 & w \\
\hline 舟 & proponomort & トセPトトトトケトト & トトトトトトトトレヒ & セトトトセ50909 & \\
\hline $\begin{array}{l}3 \\
3\end{array}$ & 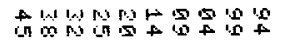 & 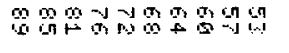 & 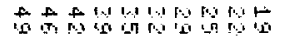 & 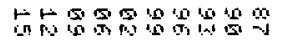 & + \\
\hline 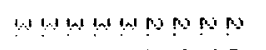 & 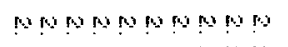 & 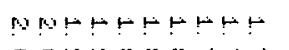 & 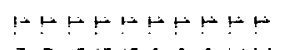 & セセトトトセトトトト & \\
\hline 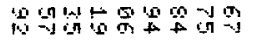 & $96+00$ & 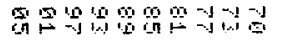 & a & 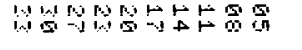 & 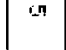 \\
\hline 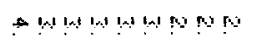 & 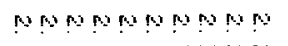 & MOONOHELL & 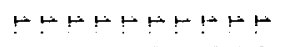 & 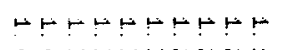 & \\
\hline 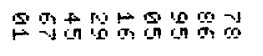 & 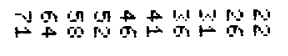 & 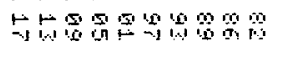 & and & 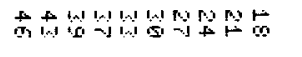 & $a$ \\
\hline F & 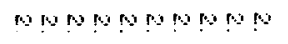 & 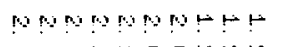 & 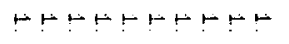 & トトトトトトケトトナ & \\
\hline 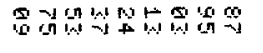 & 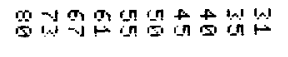 & 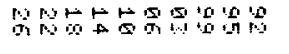 & 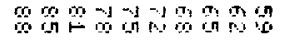 & 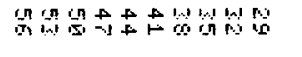 & -2 \\
\hline 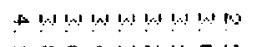 & 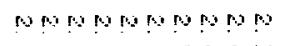 & 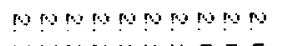 & 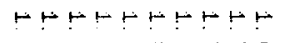 & 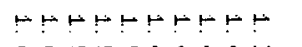 & \\
\hline 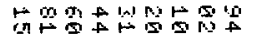 & 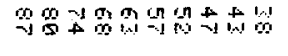 & 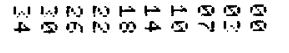 & 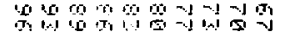 & 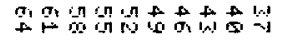 & $\infty$ \\
\hline A & 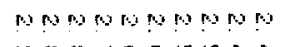 & 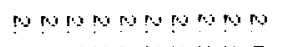 & 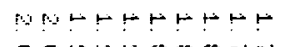 & トナトトトトトトトト & \\
\hline 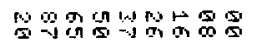 & 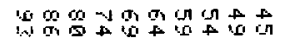 & 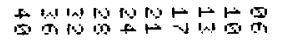 & 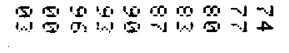 & $\begin{array}{l}2 \\
2\end{array}$ & s \\
\hline$+4 \ln 40+404$ & 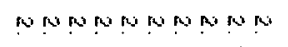 & 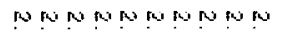 & 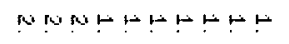 & 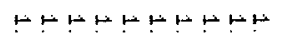 & H \\
\hline 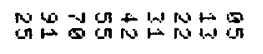 & 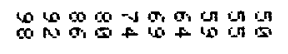 & 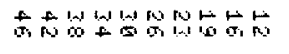 & 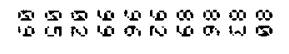 & 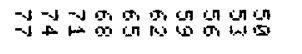 & $s$ \\
\hline F tow & 然 & 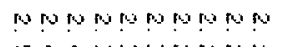 & MWWOW & 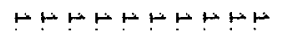 & 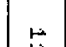 \\
\hline 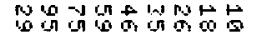 & 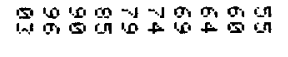 & 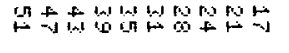 & 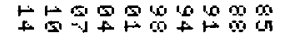 & 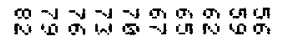 & 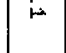 \\
\hline 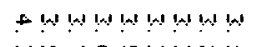 & 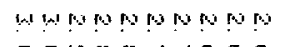 & 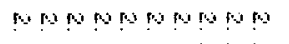 & 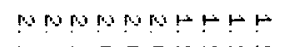 & トトトトトトトトトト & \\
\hline Who & 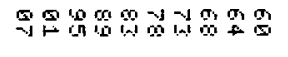 & 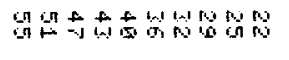 & 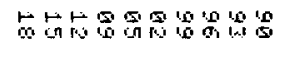 & 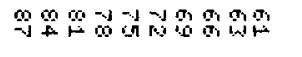 & $n$ \\
\hline 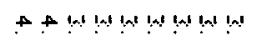 & H & 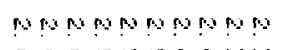 & apopopopapan & 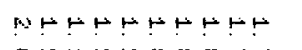 & \\
\hline 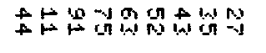 & 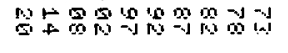 & 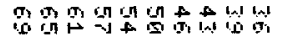 & 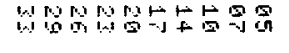 & 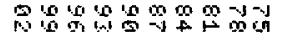 & $m$ \\
\hline 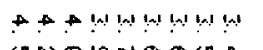 & 等 & conomonomonom & 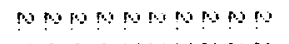 & ropononopt & $\alpha$ \\
\hline 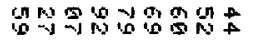 & 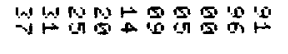 & 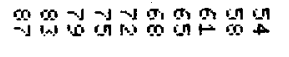 & 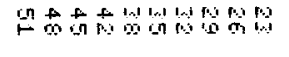 & 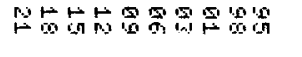 & $\leftarrow$ \\
\hline A & 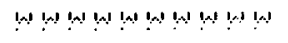 & WOONOWONOW & monomponom & 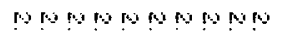 & (1) \\
\hline 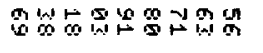 & 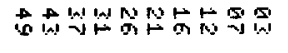 & 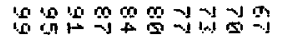 & 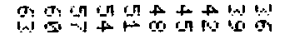 & 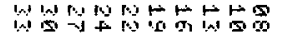 & 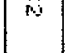 \\
\hline$n+n+\infty+\ldots+$ & 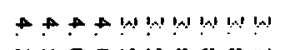 & 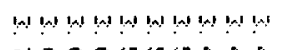 & (1) & 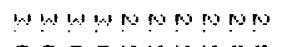 & \\
\hline 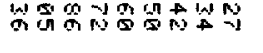 & 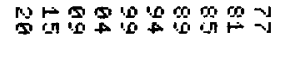 & 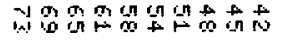 & 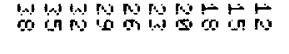 & 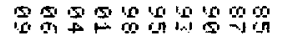 & $\stackrel{n}{n}$ \\
\hline ! $! n ! n+\infty+\infty$ & $\therefore \oplus$ & & & ant & $\stackrel{5}{5}$ \\
\hline 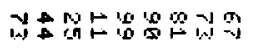 & 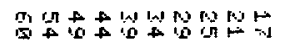 & 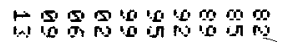 & 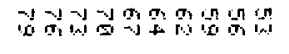 & 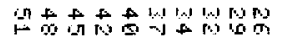 & 急 \\
\hline
\end{tabular}

